\title{
Lipid Membrane Permeability of Synthetic Redox DMPC Liposomes Investigated by Single Electrochemical Collisions
}

\author{
Estelle Lebègue,^ Frédéric Barrière,$^{\dagger}$ and Allen J. Bard ${ }^{*, \ddagger}$ \\ - Université de Nantes, CNRS, CEISAM, UMR 6230, F-44000 Nantes, France. \\ † Univ Rennes, CNRS, Institut des Sciences Chimiques de Rennes - UMR 6226, F-35000 \\ Rennes, France. \\ $\ddagger$ Center for Electrochemistry, Department of Chemistry, The University of Texas at Austin, \\ Austin, Texas 78712, United States.
}

\begin{abstract}
The electrochemical detection of synthetic redox DMPC (1,2-dimyristoyl-sn-glycero-3phosphocholine) liposomes by single collisions at $10 \mu \mathrm{m}$ diameter carbon and $\mathrm{Pt}$ ultramicroelectrodes (UMEs) is reported. To study the parameters influencing the lipid membrane opening/permeability, the electrochemical detection of single redox DMPC liposome collisions at polarized UMEs was investigated under different experimental conditions (addition of surfactant, temperature). The electrochemical responses recorded showed that the permeability of the DMPC lipid membrane (tuned by addition of Triton X-100 surfactant or by the increase of the solution temperature) is a key parameter for the liposome membrane electroporation process and hence for the release and oxidation of its redox content during the collision onto UMEs. The presence of ferrocenemethanol as an additional redox probe in the aqueous solution (at room temperature and without addition of surfactant) is also an interesting strategy to detect current spikes corresponding to single redox DMPC liposome collisions with $\mathrm{K}_{3} \mathrm{Fe}(\mathrm{CN})_{6} / \mathrm{K}_{4} \mathrm{Fe}(\mathrm{CN})_{6}$ as the encapsulated aqueous redox probe.
\end{abstract}


Electrochemical discrete collisions at an ultramicroelectrode (UME) is a useful technique to detect one at a time single biological entities such as cells, ${ }^{1-3}$ bacteria, ${ }^{4-7}$ macromolecules ${ }^{8}$ viruses, ${ }^{9-11}$ and synthetic or biological vesicles. ${ }^{12-15}$ Especially, electrochemical detection of single liposome collisions by recording electron transfer from the UME to an encapsulated redox species is fully appropriate for studying their membrane permeability. Since electron transfer do not readily occur across a bilayer lipid membrane, the electrolysis of the liposome redox active content after collision and membrane rupture or opening at the electrode surface provides insights on the membrane permeation mechanism. ${ }^{12-14,16,17}$ According to Ewing and co-workers, the so-called vesicle impact electrochemical amperometry is mainly driven by an electroporation process of the vesicle membrane on polarized carbon UMEs which leads to the vesicle rupture and the electrolysis of its content. ${ }^{18-21}$ Vesicle membrane opening by electroporation is strongly dependent on lipid membrane properties, liposome content, vesicle size, temperature, electrode potential, the nature of the electrode and probably the concentration of redox species inside and outside the liposome. ${ }^{18-22}$ For example, to observe the current spikes corresponding to the oxidation of ferrocyanide encapsulated inside phospholipid vesicles when they collide with a Pt UME, the presence of an appropriate concentration of surfactant in solution is required. ${ }^{14}$ In the absence of surfactant, we found that collision and adhesion of vesicles at the Pt UME does not allow the electrolysis of their ferrocyanide content. ${ }^{14}$ Ewing and co-workers recently hypothesized that the crucial step to initiate the membrane electroporation process of single vesicles isolated from pheochromocytoma cells (a cell line originating from adrenal chromaffin cells) is that "membrane proteins act as a barrier to electroporation and must diffuse away from the contact point between the vesicle and the electrode for membrane opening to occur". ${ }^{19}$ In addition, the osmotic pressure effects are well known to play a major role in the vesicle membrane permeability ${ }^{23-25}$ and hence the encapsulation of a high concentration of chemical redox probe such as ferrocyanide (typically $0.5 \mathrm{M}$ ) inside $100 \mathrm{~nm}$ diameter DMPC liposomes should involve changes in the membrane structure. Furthermore, it is well established that the liposome membrane stability is strongly dependent on its lipid composition and external parameters such 
as temperature and $\mathrm{pH}^{26}$ but also depends on interactions with specific molecules able to weaken, permeabilize, and penetrate the lipid bilayer following different pathways ${ }^{27-34} \mathrm{An}$ understanding of these different interactions and especially the mechanism leading to the lipid membrane opening is still an active research field. To this end, chronoamperometry is a useful method to probe the liposomes membrane permeability and to understand vesicle fusion processes onto electrode surface.$^{35-41}$ Especially, the electrochemical detection of single liposome collisions at UMEs is becoming an efficient and complementary tool for analyzing fundamental biological processes related to intracellular and extracellular electron transfers to discrete biological or artificial entities. ${ }^{42-44}$

To explore the factors influencing the vesicles membrane permeability, we investigated the electrochemical and electrocatalytic reaction of different aqueous redox species (potassium ferrocyanide and cobalt(II) nitrate) encapsulated inside synthetic DMPC $(1,2-$ dimyristoyl-sn-glycero-3-phosphocholine) liposomes subjected to different experimental conditions (addition of surfactant, increase of temperature or presence of a redox probe in solution), by single collision detection on $10 \mu \mathrm{m}$ diameter UMEs (Pt and carbon) as illustrated in Figure 1. We discuss the effect of the solution temperature on the liposome collision, its membrane rupture, and subsequent electrolysis of its redox content. We also report that the presence of an additional redox probe in the aqueous electrolyte can lead to the detection of current spikes (corresponding to the oxidation of the redox active liposomes content) in the chronoamperometric response recorded at the Pt UME. The distribution of the redox liposomes size obtained by dynamic light scattering and their size estimated from the electrochemical charge consumed during the electrolysis resulting from collision of liposomes encapsulating potassium ferrocyanide were finally compared and discussed. 

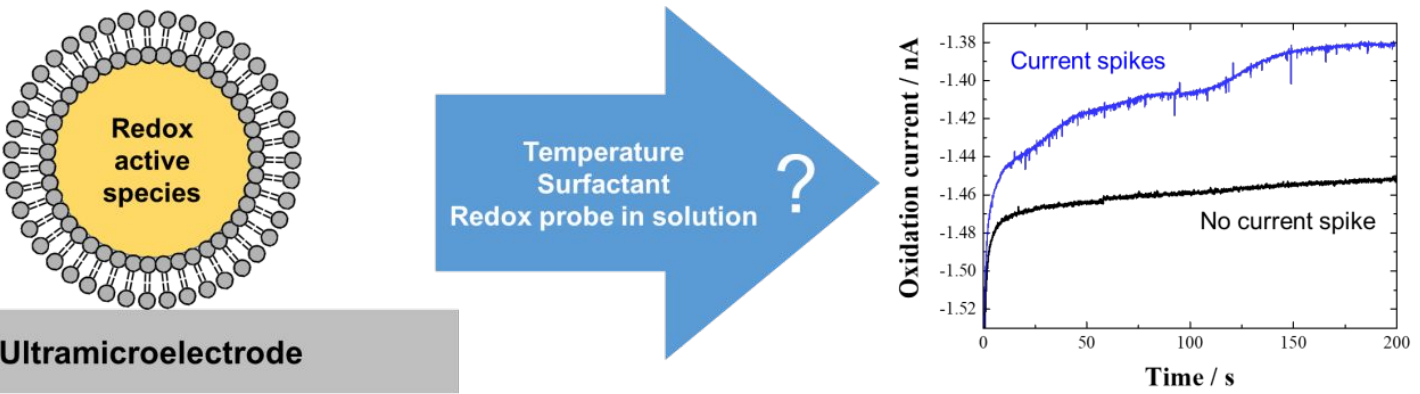

Ultramicroelectrode

Figure 1. Schematic representation of the redox DMPC liposomes collision experiments at a polarized UME in aqueous solution, and typical electrochemical responses observed in the chronoamperometric measurements. The UME is not drawn to scale.

\section{EXPERIMENTAL SECTION}

Reagents. All chemicals were reagent grade and used as purchased without further purification. Water used in each experiment was Milli-Q water. Chloroform ( $\geq 99.8 \%$ ), sulfuric acid $(97 \%)$, and hydrogen peroxide $(30 \%)$ were obtained from Fisher Scientific. Ferrocenemethanol $(97 \%)$, cobalt(II) nitrate hexahydrate $(\geq 98.0 \%)$, potassium ferrocyanide, potassium ferricyanide, potassium phosphate monobasic $(\geq 99.0 \%)$, potassium phosphate dibasic ( $\geq 98 \%$ ), and Triton X-100 were purchased from Sigma Aldrich. Pt (99.9\%) wire and carbon fiber were obtained from Goodfellow (Devon, PA). 1,2-dimyristoyl-sn-glycero-3phosphocholine (DMPC) lipids were purchased as a powder from Avanti Polar Lipids and stored in a freezer.

Liposomes preparation. Liposomes solutions were prepared by dissolving $10 \mathrm{mM}$ DMPC lipid (powder) in chloroform $(1 \mathrm{~mL})$, then vortexed for 5 minutes and placed into a warm water bath $\left(40{ }^{\circ} \mathrm{C}\right)$ for a minimum of 10 minutes until the complete dissolution of lipids. The homogeneous mixture was placed under ambient atmosphere overnight, and then under vacuum for 1 hour for the complete evaporation of chloroform. The dry lipid film was hydrated by addition of aqueous solution $(2 \mathrm{~mL}$ of potassium phosphate buffer or $2 \mathrm{~mL}$ of redox probe/catalyst aqueous solution), and then the solution was shaken for 5 minutes and heated on a hot plate at $40^{\circ} \mathrm{C}$ for 30 minutes. The DMPC liposome solutions were extruded using 400 $\mathrm{nm}$ diameter polycarbonate membranes from Avanti Polar Lipids. The liposomes solution was 
passed through the extruder 10 times, which was kept warm at $40{ }^{\circ} \mathrm{C}$, to obtain DMPC liposomes solutions. The final step was to pass DMPC liposomes solution through a column (PD-10 Desalting Columns, GE Healthcare) by using potassium phosphate buffer aqueous solution for removing redox probe/catalyst outside liposomes and typically obtaining a nanomolar range DMPC liposomes solution.

Materials and Instrumentation. The liposome extrusion was carried out with the extruder set from Avanti Polar Lipids including a mini-extruder, 2 syringes of $1 \mathrm{~mL}$, polycarbonate membranes of $0.4 \mu \mathrm{m}$, and filter supports. The electrochemical experiments were performed using a $\mathrm{CHI}$ model $920 \mathrm{C}$ and $\mathrm{CHI} 630$ potentiostat $(\mathrm{CH}$ Instruments, Austin, TX) with a three-electrode cell placed in a Faraday cage and using the $\mathrm{CHI}$ Instruments software. Pt wire was used as a counter electrode, and the reference electrode was $\mathrm{Ag} / \mathrm{AgCl}$ (3 $\mathrm{M} \mathrm{KCl}$ ). For all chronamperometric i-t curves recorded, the sample interval (in sampling time) was $50 \mathrm{~ms}$ and the signal filter used was $150 \mathrm{~Hz}$. Dynamic light scattering data was carried out on a Zetasizer Nano ZS (Malvern, Westborough, MA). All data and results presented in the manuscript and Supporting Information are reproducible and were repeated at least three times in the same experimental conditions. Note that the electrochemical measurements at different temperatures (higher level of noise) consistently report that there is an optimum temperature $\left(60 \pm 5^{\circ} \mathrm{C}\right)$ for which a maximum frequency of release events is observed. At this optimum temperature (experimentally either 55 or $60{ }^{\circ} \mathrm{C}$ ) the maximum frequency only changes by a factor of 2 (Figure S2).

The carbon fiber and Pt ultramicroelectrodes (UMEs) were prepared following a published procedure. ${ }^{45,46}$ In short, UMEs were prepared by sealing a $10 \mu \mathrm{m}$ diameter carbon fiber or $\mathrm{Pt}$ wire in a borosilicate capillary using resistive heating. Silver epoxy was used to connect the electrode to a nickel-chromium wire. The electrode was then polished to expose the active surface of the carbon or that of platinum. Before each experiment, electrodes were mechanically polished using wetted diamond polishing pads and washed successively in water, acetone, ethanol, and several times in water. The ultramicroelectrode was then immersed in the electrolyte, connected as a working electrode and the chronoamperometric 
measurement at the required potential was launched straight away in a matter of a few seconds (max. $5 \mathrm{~s}$ of elapsed time). After injection of redox liposomes and/or surfactant solution in the electrochemical cell, the electrolyte was let to stabilize for 5 min before immersing the ultramicroelectrode and launching the chronoamperometry.

\section{RESULTS AND DISCUSSION}

We have previously demonstrated that the presence of an appropriate concentration of surfactant $(0.20 \pm 0.03 \mathrm{mM}$ of Triton $\mathrm{X}-100)$ close to the critical micelle concentration $(\mathrm{CMC}=$ $0.17 \mathrm{mM})^{47}$ in aqueous solution of redox DMPC liposomes encapsulating potassium ferrocyanide as a redox probe is necessary for weakening their lipid membrane and to observe single electrochemical collisions at Pt UMEs. ${ }^{14}$ Previous investigations of vesicle adsorption on different substrates have shown that vesicles adsorbed on hydrophilic surfaces such as $\mathrm{Pt}$ electrodes tend to remain intact while they are prone to break (membrane rupture) on hydrophobic surfaces. ${ }^{48,49}$ Herein, we first study the effects of the encapsulated redox probe on liposome membrane permeability. Chronoamperometric measurements were carried out at a polarized $10 \mu \mathrm{m}$ diameter carbon UME (+1.2 V vs. $\mathrm{Ag} / \mathrm{AgCl})$ in potassium phosphate buffer as aqueous electrolyte in the presence of DMPC liposomes that contained cobalt(II) nitrate as catalyst for water oxidation ${ }^{50}$ (Figure 2). For DMPC liposomes, addition of an appropriate concentration of Triton $\mathrm{X}-100$ (previously optimized at $0.2 \mathrm{mM})^{14}$ as a surfactant in the electrolyte is required to detect current spikes (orange i-t curve in Figure 2) corresponding to the liposome membrane breaking and the cobalt(II) content release catalyzing water oxidation at this potential during collision on the carbon electrode surface. Because of the short elapsed time (max. $5 \mathrm{~s}$ ) between the ultramicroelectrode immersion and the launching of the chronoamperometric measurement, pre-adsorption of the liposomes can be excluded in these experimental conditions and the observed current spikes can be confidently assigned to collision and rupture of redox liposomes. The need to add surfactant here is at variance with other reports on different liposomes where the observation of current spikes at a carbon UME does not require any additive..$^{15,17,19}$ This observation coupled to those from our previous work ${ }^{14}$ 
suggest that irrespective of the nature of the encapsulated redox probe and its concentration, the nature of the electrode or the applied potential, the lipid membrane of synthetic redox DMPC liposomes is robust enough to withstand collision on the carbon UME surface (blue $i-t$ curve in Figure 2). The relatively more robust and stable lipid bilayer of pure DMPC liposomes studied in the present work is most likely the reason for the apparent discrepancy with other works, ${ }^{15,17-19}$ for example those of Ewing and co-workers ${ }^{19}$ who used pheochromocytoma cells with a complex glycosphingolipid membrane composition ${ }^{51}$ or liposomes composed of lipid mixture (DOPC/DOPE/cholesterol) which are less robust in their experimental conditions (37 $\left.{ }^{\circ} \mathrm{C}\right) .{ }^{18}$ In addition, the DMPC liposomes size (here the hydrodynamic liposome diameter estimated from dynamic light scattering data is ca. $250 \mathrm{~nm}$, Figure S1) does not change the lipid membrane permeability as these experiments have been repeated for liposomes with diameter in the range 100 to $300 \mathrm{~nm}$. This result confirms that synthetic redox liposomes based on a pure DMPC lipid bilayer do not break during collision onto a platinum or carbon UME surface at $20^{\circ} \mathrm{C}$ and that an external stimulus such as a surfactant (Triton $\mathrm{X}-100$ ) is required to permeate the lipid membrane and bring about liposome lysis.
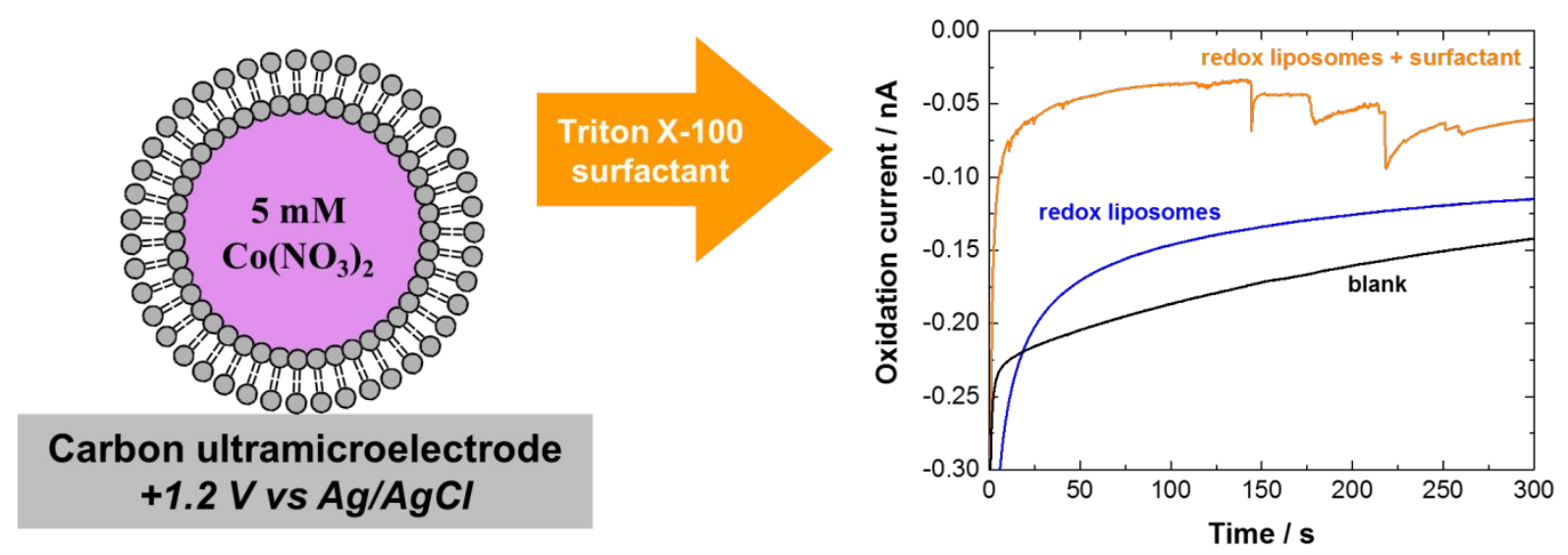

Figure 2. Schematic representation and the $i-t$ curve for collision experiments of redox DMPC liposomes encapsulating $5 \mathrm{mM}$ cobalt(II) nitrate recorded at a $10 \mu \mathrm{m}$ diameter carbon UME polarized at $+1.2 \mathrm{~V} v \mathrm{vs}$. $\mathrm{Ag} / \mathrm{AgCl}$ in $2 \mathrm{~mL}$ of $0.1 \mathrm{M}$ potassium phosphate buffer aqueous solution at $\mathrm{pH} 7$ in the absence (black) and in the presence of $20 \mu \mathrm{L}$ of redox DMPC liposomes aqueous solution with (orange) and without (blue) addition of $0.2 \mathrm{mM}$ Triton X-100 surfactant. Temperature: $20^{\circ} \mathrm{C}$. The UME is not drawn to scale. 
We also investigated the effect of increasing the temperature of the liposomes solution on the lipid membrane permeability and the detection of single collisions. The chronoamperometric measurements presented in Figure 3 were performed at a polarized $10 \mu \mathrm{m}$ diameter Pt UME $(+0.6 \mathrm{~V} v \mathrm{vs} . \mathrm{Ag} / \mathrm{AgCl})$ in potassium phosphate buffer at $\mathrm{pH} 7$ as aqueous electrolyte at different temperatures (from 20 to $70{ }^{\circ} \mathrm{C}$ ) in the presence of synthetic DMPC liposomes encapsulating potassium ferrocyanide $\left(\left[\mathrm{K}_{4} \mathrm{Fe}(\mathrm{CN})_{6}\right]=0.5 \mathrm{M}, \mathrm{E}^{0}=+0.20 \mathrm{~V} v \mathrm{vs} . \mathrm{Ag} / \mathrm{AgCl}\right)$ as redox probe. The lipid membrane permeability is strongly dependent on the temperature, and there is an optimal temperature where this permeability is maximum depending on the lipid membrane composition..$^{52,53}$ The $i-t$ curves reported in Figure 3 show that current spikes corresponding to the release of the redox DMPC liposomes content during single collisions on a Pt UME are detected when the temperature reaches $45^{\circ} \mathrm{C}$ (the pink i-t curve in Figure 3 ). The frequency of release events then increases from $0.01 \pm 0.01 \mathrm{~Hz}$ at $45 \pm 5{ }^{\circ} \mathrm{C}$ up to a maximum value of $0.12 \pm 0.08 \mathrm{~Hz}$ at $60 \pm 5^{\circ} \mathrm{C}$ (Figures 3 and S2, Table S1). Note that the experimental frequency of release events is always found lower than the theoretical liposome collision frequency (calculated using either a steady state or transient diffusion model). This maybe rationalized by the fact that above $50{ }^{\circ} \mathrm{C}$ there are some redox liposomes that immediately break upon impact (observation of release events), others that only adsorb without membrane rupture (no release event) or that release their redox content in solution without collision (for the most weakened membranes). The small difference $( \pm 0.03 \mathrm{~Hz})$ reported in Table S1 between the theoretical collision frequency values determined with either a steady-state or a transient model confirms that both models are appropriate for the time scale of our experiments (300 s). Taken together these results show that varying the temperature is an efficient external stimulus for weakening the membrane and observing the release events. The maximum collision frequency temperature $\left(60 \pm 5^{\circ} \mathrm{C}\right)$ is slightly but significantly higher than those reported for the maximum membrane permeability of various phospholipid liposomes $\left(\sim 42^{\circ} \mathrm{C}\right),{ }^{52-54}$ and clearly higher than the phase transition temperature of the DMPC lipid $\left(24^{\circ} \mathrm{C}\right)$. Assuming that the concentration of encapsulated ferrocyanide $(0.5 \mathrm{M})$ is the same in each redox liposome and 
that all the liposome content is electrolyzed during the collision onto the UME, integration of the total charge transferred during each current spike allows the calculation of the liposome hydrodynamic diameter using Faraday's law. The mean diameter of the liposomes $(270 \mathrm{~nm} \pm$ $52 \%$ ) estimated from the charge passed during collisions (Figure S4) is close to the average value obtained from dynamic light scattering data (Figure S3) indicating that the current spikes observed in the orange $i-t$ curve in Figure $3\left(55^{\circ} \mathrm{C}\right)$ are due to oxidation of the ferrocyanide content of single liposomes. Importantly, over the time scale of the experiment (max. $300 \mathrm{~s}$ ) the size distribution of redox liposomes (136 to $403 \mathrm{~nm}$ ) estimated by charge integration of the current spikes is consistent with those determined by dynamic light scattering data in the first Gaussian peak centered at $240 \pm 67 \mathrm{~nm}$ and representing more than $80 \%$ of particles in solution (Figure S3). Because a "signal" (collision) is considered when the current spike is at least three times the background current noise, the smaller redox liposomes $(<120 \mathrm{~nm}$ of mean diameter) are not detected in the electrochemical measurement at the optimal temperature of $60 \pm 5^{\circ} \mathrm{C}$, with a limit of detection corresponding to an integrated charge of $100 \mathrm{fC}$. In addition, the current spikes observed at $55^{\circ} \mathrm{C}$ are higher and narrower than those observed at $45^{\circ} \mathrm{C}$ (Figure S5), meaning that a lower temperature results in a longer release of the liposome redox content upon collision. This phenomenon is probably related to the weakened level of the liposome membrane. Indeed, at lower temperature, the liposome membrane is less weakened and hence a longer release is observed (longer electrolysis time) due to gradual and longer partial membrane opening. In contrast, at higher temperature the redox liposome is more weakened and hence immediately broken upon collision onto the ultramicroelectrode, leading to a fast electrolysis. Above $60{ }^{\circ} \mathrm{C}$, the noise level in the chronoamperometric curves (Figure 3 ) is too high to clearly discriminate the signal of the collision events as confirmed by the control experiments reported in Figure S6. These results demonstrate the robustness of the homemade synthetic redox liposomes of pure DMPC with a lipid membrane that only starts to weaken above $40^{\circ} \mathrm{C}$. The effect of temperature is similar to the effect of surfactant previously studied on the liposomes membrane permeability showing that this parameter can also be an efficient external stimulus (in place of/or coupled to the addition of a surfactant) for detection 
of current spikes corresponding to the liposomes redox probe electrolysis upon impact at the electrode surface.

A
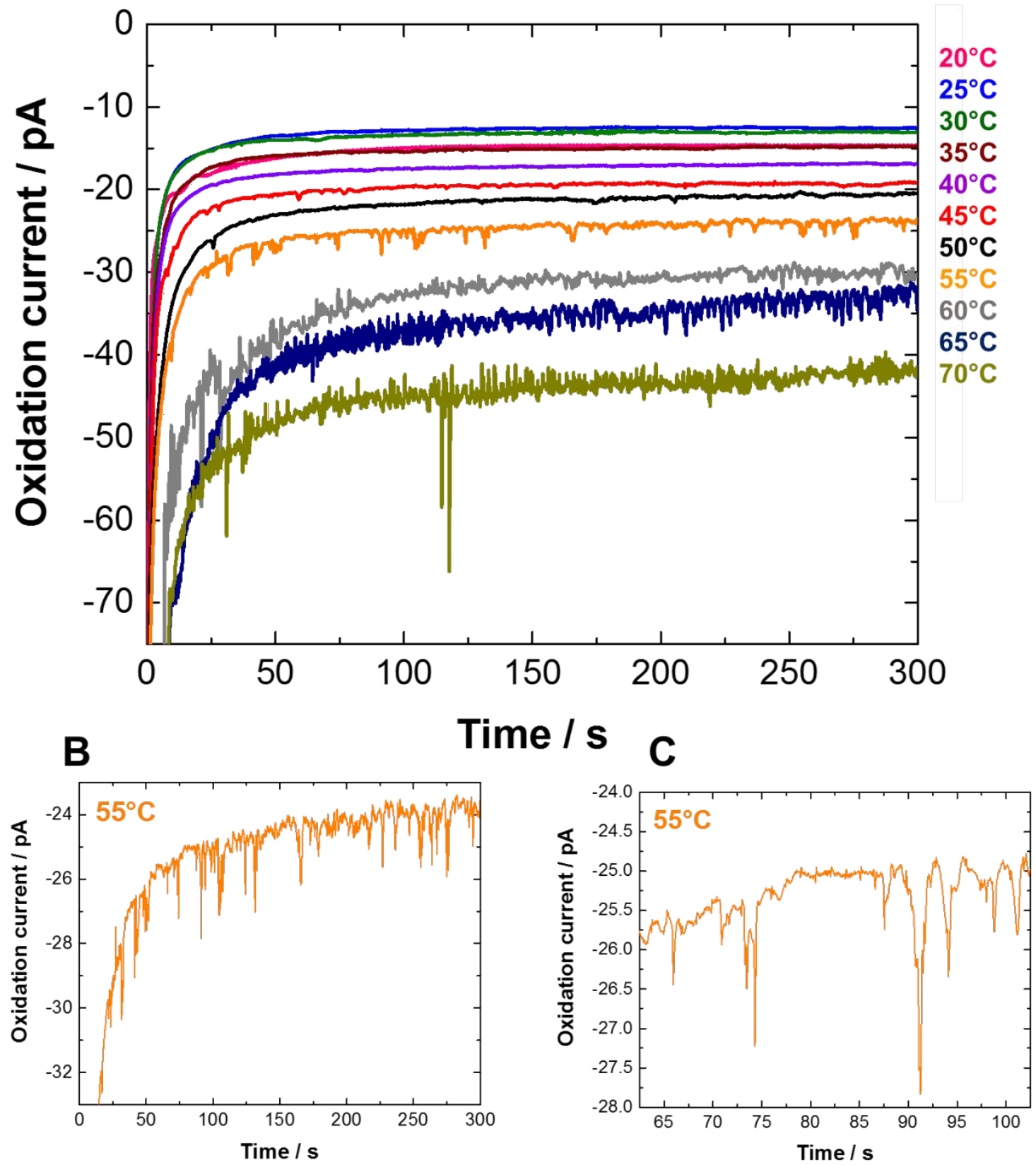

Figure 3.The $i$ - $t$ curve for collision experiments recorded at $+0.6 \mathrm{~V} v \mathrm{~s}$. $\mathrm{Ag} / \mathrm{AgCl}$ on $10 \mu \mathrm{m}$ diameter Pt UME in $30 \mathrm{~mL}$ of $0.1 \mathrm{M}$ potassium phosphate buffer aqueous solution at $\mathrm{pH} 7$ in the presence of $0.6 \mathrm{~mL}$ of redox DMPC liposomes aqueous solution at different temperatures (from 20 to $70{ }^{\circ} \mathrm{C}$ ). Figures $3 \mathrm{~B}$ and $3 \mathrm{C}$ are enlarged portions of the orange $i$ - $t$ curve $\left(55^{\circ} \mathrm{C}\right)$ of Figure 3A. 
While studying different parameters affecting the lipid bilayer permeability of redox liposomes, we also observed the interesting effect of adding a redox probe $\left(1 \mathrm{mM}\right.$ ferrocenemethanol, $\mathrm{E}^{0}$ $=+0.15 \mathrm{~V}$ vs. $\mathrm{Ag} / \mathrm{AgCl}$ ) in the aqueous solution containing redox DMPC liposomes (with encapsulated $0.5 \mathrm{M} \mathrm{K}_{3} \mathrm{Fe}(\mathrm{CN})_{6} / 0.5 \mathrm{M} \mathrm{K}_{4} \mathrm{Fe}(\mathrm{CN})_{6}, \mathrm{E}^{0}=+0.20 \mathrm{~V}$ vs. Ag/AgCl). Indeed, the addition of a redox probe in potassium phosphate buffer aqueous solution containing redox DMPC liposomes leads to single collisions events at Pt UME. Thus, in the absence of surfactant and at room temperature $\left(20^{\circ} \mathrm{C}\right)$, the electrochemical detection of collisions of single redox DMPC liposome containing an equimolar amount of ferri/ferrocyanide at a $10 \mu \mathrm{m} \mathrm{Pt}$ UME polarized at $+0.3 \mathrm{~V} v s$. $\mathrm{Ag} / \mathrm{AgCl}$ (an oxidizing potential for both ferrocenemethanol and ferrocyanide) was successfully achieved in a $1 \mathrm{mM}$ ferrocenemethanol aqueous solution (Figure 4). Note that the presence of both ferrocyanide and ferricyanide in the liposome is required to detect current spikes at Pt UME corresponding to the oxidation of ferrocyanide at the applied electrode potential $(+0.3 \mathrm{~V})$. Control experiments with only ferrocyanide encapsulated in the liposomes demonstrate the absence of current spikes (see Figure S7A for a representative example). In addition, we have checked that in the absence of ferrocenemethanol, liposomes containing both ferro- and ferricyanide do not undergo electrolysis of their content (see Figure S7B for a representative example). The mean hydrodynamic diameter of redox DMPC liposomes was estimated using Faraday's law, and the integration of the charge consumed during each collision in the current spikes corresponding to the ferrocyanide oxidation (the orange $i$-t curve in Figure 4). This estimated diameter is in agreement with the dynamic light scattering data (Figure S8). This result shows that the presence of ferrocenemethanol in aqueous solution containing DMPC liposomes encapsulating $\mathrm{K}_{3} \mathrm{Fe}(\mathrm{CN})_{6} / \mathrm{K}_{4} \mathrm{Fe}(\mathrm{CN})_{6}$ as redox probe is necessary and sufficient to observe current spikes recorded at Pt UME polarized at $+0.3 \mathrm{~V}$ (the orange $i-t$ curve in Figure 4). This observation suggests that the $(\mathrm{FcMeOH})^{+} / \mathrm{FcMeOH}$ chemical species, relatively soluble in water but possessing some lipophilicity, ${ }^{55,56}$ play the role of redox mediators or increase the lipid membrane permeability, and hence facilitates electron transfer between the liposome 
content and the UME after impact, possibly without full membrane breaking. This hypothesis is supported by the observation of current spikes corresponding to single redox liposome collisions only when the UME is polarized at potentials sufficiently oxidizing for ferrocenemethanol (> $0.2 \mathrm{~V}$ vs. $\mathrm{Ag} / \mathrm{AgCl}$, Figure S9). Comparison of the estimation of liposomes diameter from collisions experiments $(230 \mathrm{~nm} \pm 77 \%)$ with the size distribution determined by dynamic light scattering ( $\sim 280 \mathrm{~nm}$, Figure S8) leads to the conclusion that the liposomes ferrocyanide redox content is not entirely oxidized. Thus, only a partial electrolysis of ferrocyanide $(\sim 80 \%)$ occurs during the collision onto the UME. This observation shows that the process occurring here for oxidation of the redox liposomes content is different than the transfection mechanism previously proposed for the membrane permeabilization with the nonionic surfactant Triton $\mathrm{X}-100 .{ }^{14,47,57}$ Contrary to the surfactant and temperature effects discussed above that induce electroporation and fusion processes of weakened redox DMPC liposomes on the UME surface, the addition of ferrocenemethanol as a redox probe in solution only leads to a partial electrolysis by a different mechanism possibly involving the lipophilic and/or redox shuttle properties of $(\mathrm{FcMeOH})^{+} / \mathrm{FcMeOH}$ with no associated full membrane rupture. This different suggested role is also supported by the comparison of the shape of the current spikes in other experimental conditions. In the presence of ferrocenemethanol and redox liposomes containing ferri/ferrocyanide (orange i-t curve, Figure 4) the current spikes show significantly lower half-time $(0.15 \mathrm{~s})$ and rise time $(0.13 \mathrm{~s})$ compared for example with those reported in Figure 3 (orange i-t curve) for which we find half-time of $0.33 \mathrm{~s}$ and rise time of $0.35 \mathrm{~s}$. Additionally, previous data ${ }^{14}$ also show comparatively higher half- and rise time $(0.58 / 0.21 \mathrm{~s}$ respectively). This suggests a different mechanism in the presence of ferrocenemethanol and lends supports to a redox shuttle behavior (comparatively partial and rapid electrolysis of the liposome content upon collision) rather than a surfactant behavior that would cause complete and longer electrolysis upon membrane weakening and rupture. This novel and non-destructive mechanism should be clarified and extended to other aqueous redox active probes with different normal potential and hydrophilic/hydrophobic properties and to other artificial or natural liposomes/vesicles. 


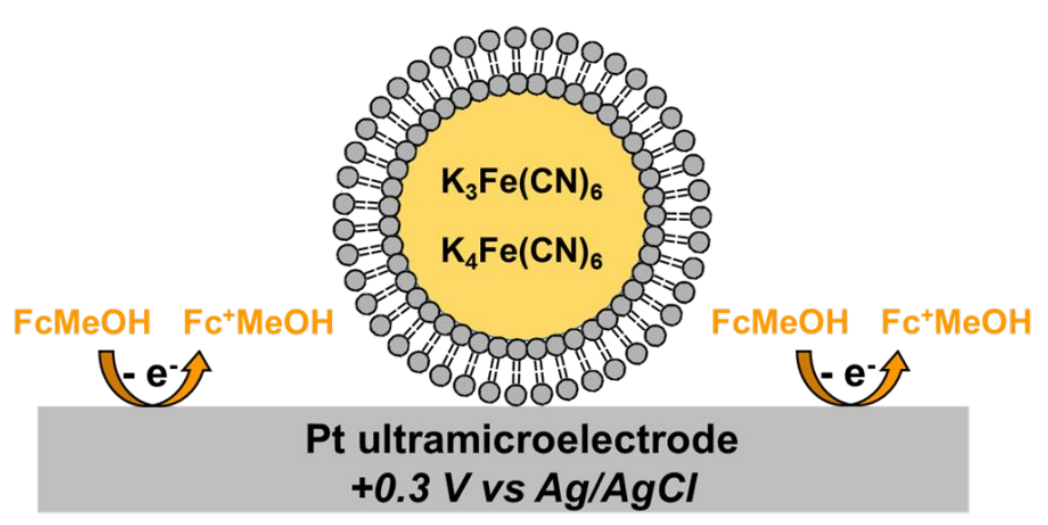

A

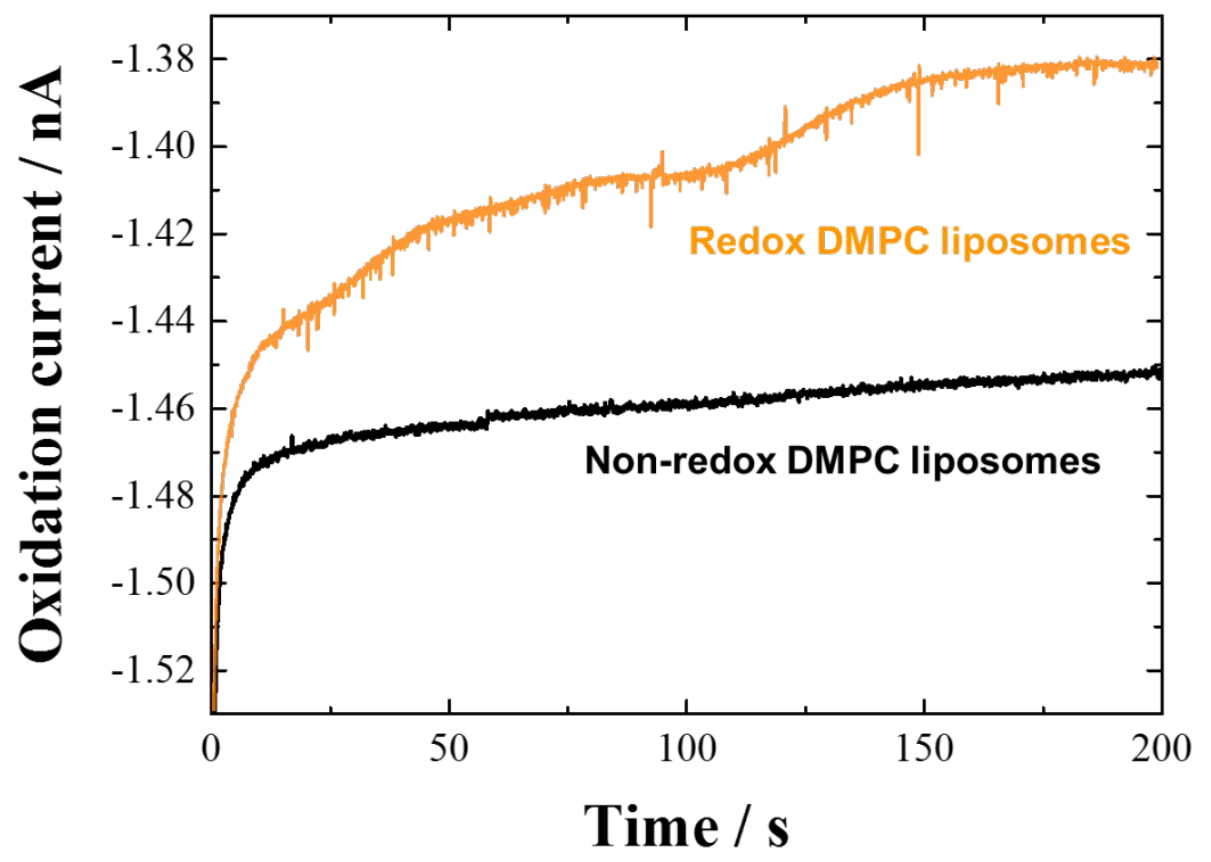

B

C
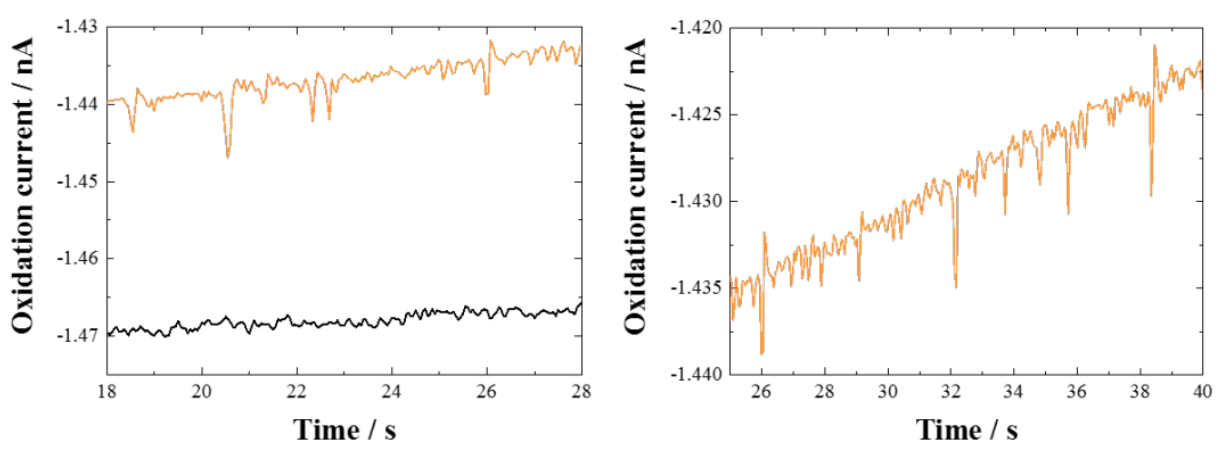

Figure 4. Schematic representation and the $i$ - $t$ curve for collision experiments recorded at +0.3 V vs. $\mathrm{Ag} / \mathrm{AgCl}$ on $10 \mu \mathrm{m} \mathrm{Pt}$ UME in $2 \mathrm{~mL}$ of $1 \mathrm{mM}$ ferrocenemethanol aqueous solution in the presence of $100 \mu \mathrm{L}$ non-redox DMPC liposomes (black curve) and redox DMPC liposomes (orange curve) aqueous solution. Figures $4 B$ and $4 C$ are enlarged portions of Figure 4A. Temperature: $20^{\circ} \mathrm{C}$. The UME is not drawn to scale. 


\section{CONCLUSIONS}

In summary, the electrochemical detection of synthetic redox DMPC liposomes by single collisions at a carbon or Pt UME under different experimental conditions has been successfully achieved. The influence of the presence of surfactant (Triton X-100) and the increase of solution temperature $\left(20-70{ }^{\circ} \mathrm{C}\right)$ on the lipid membrane permeability has been investigated, and the results showed a similar effect of these two parameters with a maximum collision frequency reached for an optimal surfactant concentration $(0.2 \mathrm{mM})$ and temperature $\left(60^{\circ} \mathrm{C}\right)$ respectively, at platinum or carbon UMEs. An interesting result has also been reported showing that the presence of ferrocenemethanol as an additional redox probe dissolved in the liposomes aqueous solution at $20{ }^{\circ} \mathrm{C}$, allows observation of current spikes in the chronoamperometric curve. This corresponds to partial oxidation of the liposomes redox content $\left(\mathrm{K}_{4} \mathrm{Fe}(\mathrm{CN})_{6} / \mathrm{K}_{3} \mathrm{Fe}(\mathrm{CN})_{6}\right)$ during collision on UME surface and is only observed provided that the applied potential is greater than that of $(\mathrm{FcMeOH})^{+} / \mathrm{FcMeOH}$. This last result opens the way to electron shuttling and/or membrane permeabilization via a redox species able to diffuse through or dissolve in the thick lipid membranes of redox liposomes. Observation of single electrochemical collisions is a powerful method to study liposomes lipid membrane permeability and various interaction mechanisms which influences its stability and its structure. This study can be extended to other aqueous redox active probes and to biomolecules able to interact with a cell's lipid membrane for a better understanding of fundamental biological processes.

\section{ASSOCIATED CONTENT}

The Supporting Information is available free of charge on the ACS Publications website. Dynamic light scattering data; data processing (collision frequency and charge integration) from collision experiments.

\section{AUTHOR INFORMATION}




\section{Corresponding Author}

*E-mail: ajbard@cm.utexas.edu

ORCID

Frédéric Barrière: 0000-0001-5515-4080

Allen J. Bard: 0000-0002-8517-0230

\section{Notes}

The authors declare no competing financial interest.

\section{ACKNOWLEDGMENTS}

A.J.B. acknowledges the National Science Foundation (Grant Nos. CHE-1405248 and CHE1707384) and the Welch Foundation (Grant No. F-0021).

\section{REFERENCES}

(1) Dick, J. E. Electrochemical Detection of Single Cancer and Healthy Cell Collisions on a Microelectrode. Chem. Commun. 2016, 52 (72), 10906-10909. https://doi.org/10.1039/C6CC04515D.

(2) Gooding, J. J. Single Entity Electrochemistry Progresses to Cell Counting. Angew. Chem. Int. Ed. 2016, 55 (42), 12956-12958. https://doi.org/10.1002/anie.201606459.

(3) Sepunaru, L.; Sokolov, S. V.; Holter, J.; Young, N. P.; Compton, R. G. Electrochemical Red Blood Cell Counting: One at a Time. Angew. Chem. Int. Ed. 2016, 55 (33), 97689771. https://doi.org/10.1002/anie.201605310.

(4) Sepunaru, L.; Tschulik, K.; Batchelor-McAuley, C.; Gavish, R.; Compton, R. G. Electrochemical Detection of Single E. Coli Bacteria Labeled with Silver Nanoparticles. Biomater. Sci. 2015, 3 (6), 816-820. https://doi.org/10.1039/C5BM00114E.

(5) Lee, J. Y.; Kim, B.-K.; Kang, M.; Park, J. H. Label-Free Detection of Single Living Bacteria via Electrochemical Collision Event. Sci. Rep. 2016, 6, 30022. https://doi.org/10.1038/srep30022.

(6) Ronspees, A. T.; Thorgaard, S. N. Blocking Electrochemical Collisions of Single E. Coli and B. Subtilis Bacteria at Ultramicroelectrodes Elucidated Using Simultaneous Fluorescence Microscopy. Electrochimica Acta 2018, 278, 412-420. https://doi.org/10.1016/j.electacta.2018.05.006.

(7) Gao, G.; Wang, D.; Brocenschi, R.; Zhi, J.; Mirkin, M. V. Toward the Detection and Identification of Single Bacteria by Electrochemical Collision Technique. Anal. Chem. 2018. https://doi.org/10.1021/acs.analchem.8b03043.

(8) Dick, J. E.; Renault, C.; Bard, A. J. Observation of Single-Protein and DNA Macromolecule Collisions on Ultramicroelectrodes. J. Am. Chem. Soc. 2015, 137 (26), 8376-8379. https://doi.org/10.1021/jacs.5b04545.

(9) Dick, J. E.; Hilterbrand, A. T.; Boika, A.; Upton, J. W.; Bard, A. J. Electrochemical Detection of a Single Cytomegalovirus at an Ultramicroelectrode and Its Antibody 
Anchoring. Proc. Natl. Acad. Sci. 2015, 112 (17), 5303-5308. https://doi.org/10.1073/pnas.1504294112.

(10) Dick, J. E.; Hilterbrand, A. T.; Strawsine, L. M.; Upton, J. W.; Bard, A. J. Enzymatically Enhanced Collisions on Ultramicroelectrodes for Specific and Rapid Detection of Individual Viruses. Proc. Natl. Acad. Sci. 2016, 113 (23), 6403-6408. https://doi.org/10.1073/pnas.1605002113.

(11) Sepunaru, L.; Plowman, B. J.; Sokolov, S. V.; Young, N. P.; Compton, R. G. Rapid Electrochemical Detection of Single Influenza Viruses Tagged with Silver Nanoparticles. Chem. Sci. 2016, 7 (6), 3892-3899. https://doi.org/10.1039/C6SC00412A.

(12) Cheng, W.; Compton, R. G. Investigation of Single-Drug-Encapsulating Liposomes Using the Nano-Impact Method. Angew. Chem. Int. Ed. 2014, 53 (50), 13928-13930. https://doi.org/10.1002/anie.201408934.

(13) Dunevall, J.; Fathali, H.; Najafinobar, N.; Lovric, J.; Wigström, J.; Cans, A.-S.; Ewing, A. G. Characterizing the Catecholamine Content of Single Mammalian Vesicles by Collision-Adsorption Events at an Electrode. J. Am. Chem. Soc. 2015, 137 (13), 43444346. https://doi.org/10.1021/ja512972f.

(14) Lebègue, E.; Anderson, C. M.; Dick, J. E.; Webb, L. J.; Bard, A. J. Electrochemical Detection of Single Phospholipid Vesicle Collisions at a $\mathrm{Pt}$ Ultramicroelectrode. Langmuir 2015, 31 (42), 11734-11739. https://doi.org/10.1021/acs.langmuir.5b03123.

(15) Zhang, Y.; Li, M.; Li, Z.; Li, Q.; Aldalbahi, A.; Shi, J.; Wang, L.; Fan, C.; Zuo, X. Recognizing Single Phospholipid Vesicle Collisions on Carbon Fiber Nanoelectrode. Sci. China Chem. 2017, 60 (11), 1474-1480. https://doi.org/10.1007/s11426-017-90360.

(16) Dunevall, J.; Majdi, S.; Larsson, A.; Ewing, A. Vesicle Impact Electrochemical Cytometry Compared to Amperometric Exocytosis Measurements. Curr. Opin. Electrochem. 2017, 5 (1), 85-91. https://doi.org/10.1016/j.coelec.2017.07.005.

(17) Cheng, W.; Compton, R. G. Measuring the Content of a Single Liposome through Electrocatalytic Nanoimpact "Titrations." ChemElectroChem 2016, 3 (12), 2017-2020. https://doi.org/10.1002/celc.201600396.

(18) Lovrić, J.; Najafinobar, N.; Dunevall, J.; Majdi, S.; Svir, I.; Oleinick, A.; Amatore, C.; Ewing, A. G. On the Mechanism of Electrochemical Vesicle Cytometry: Chromaffin Cell Vesicles and Liposomes. Faraday Discuss. 2016, 193 (0), 65-79. https://doi.org/10.1039/C6FD00102E.

(19) Li, X.; Dunevall, J.; Ren, L.; Ewing, A. G. Mechanistic Aspects of Vesicle Opening during Analysis with Vesicle Impact Electrochemical Cytometry. Anal. Chem. 2017, 89 (17), 9416-9423. https://doi.org/10.1021/acs.analchem.7b02226.

(20) Li, X.; Ren, L.; Dunevall, J.; Ye, D.; White, H. S.; Edwards, M. A.; Ewing, A. G. Nanopore Opening at Flat and Nanotip Conical Electrodes during Vesicle Impact Electrochemical Cytometry. ACS Nano 2018, $12 \quad$ (3), 3010-3019. https://doi.org/10.1021/acsnano.8b00781.

(21) Najafinobar, N.; Lovrić, J.; Majdi, S.; Dunevall, J.; Cans, A.-S.; Ewing, A. Excited Fluorophores Enhance the Opening of Vesicles at Electrode Surfaces in Vesicle Electrochemical Cytometry. Angew. Chem. Int. Ed. 2016, 55 (48), 15081-15085. https://doi.org/10.1002/anie.201609178.

(22) Li, X.; Dunevall, J.; Ewing, A. G. Quantitative Chemical Measurements of Vesicular Transmitters with Electrochemical Cytometry. Acc. Chem. Res. 2016, 49 (10), 23472354. https://doi.org/10.1021/acs.accounts.6b00331.

(23) Miller, C.; Arvan, P.; Telford, J. N.; Racker, E. Ca++-Induced Fusion of Proteoliposomes: Dependence on Transmembrane Osmotic Gradient. J. Membr. Biol. 1976, 30 (1), $271-$ 282. https://doi.org/10.1007/bf01869672.

(24) Surewicz, W. K. Effect of Osmotic Gradient on the Physical Properties of Membrane Lipids in Liposomes. Chem. Phys. Lipids 1983, 33 (1), 81-85. https://doi.org/10.1016/0009-3084(83)90010-5.

(25) White, G. F.; Racher, K. I.; Lipski, A.; Hallett, F. R.; Wood, J. M. Physical Properties of Liposomes and Proteoliposomes Prepared from Escherichia Coli Polar Lipids. Biochim. 
Biophys. Acta BBA - Biomembr. 2000, 1468 (1), 175-186. https://doi.org/10.1016/S0005-2736(00)00255-8.

(26) Sułkowski, W. W.; Pentak, D.; Nowak, K.; Sułkowska, A. The Influence of Temperature, Cholesterol Content and PH on Liposome Stability; 2005; Vol. 744. https://doi.org/10.1016/j.molstruc.2004.11.075.

(27) Xu, D.; Cheng, Q. Surface-Bound Lipid Vesicles Encapsulating Redox Species for Amperometric Biosensing of Pore-Forming Bacterial Toxins. J. Am. Chem. Soc. 2002, 124 (48), 14314-14315. https://doi.org/10.1021/ja027897f.

(28) Ciobanasu, C.; Dragomir, I.; Apetrei, A. The Penetrating Properties of the Tumor Homing Peptide LyP-1 in Model Lipid Membranes. J. Pept. Sci. 2019, 25 (3), e3145. https://doi.org/10.1002/psc.3145.

(29) Mahendra, A.; James, H. P.; Jadhav, S. PEG-Grafted Phospholipids in Vesicles: Effect of PEG Chain Length and Concentration on Mechanical Properties. Chem. Phys. Lipids 2019, 218, 47-56. https://doi.org/10.1016/j.chemphyslip.2018.12.001.

(30) Zylberberg, C.; Matosevic, S. Pharmaceutical Liposomal Drug Delivery: A Review of New Delivery Systems and a Look at the Regulatory Landscape. Drug Deliv. 2016, 23 (9), 3319-3329. https://doi.org/10.1080/10717544.2016.1177136.

(31) Brander, S.; Jank, T.; Hugel, T. AFM Imaging Suggests Receptor-Free Penetration of Lipid Bilayers by Toxins. Langmuir 2019, 35 (2), 365-371. https://doi.org/10.1021/acs.langmuir.8b03146.

(32) Nasir, M. Z. M.; Jackman, J. A.; Cho, N.-J.; Ambrosi, A.; Pumera, M. Detection of Amphipathic Viral Peptide on Screen-Printed Electrodes by Liposome Rupture Impact Voltammetry. Anal. Chem. 2017, 89 (21), 11753-11757. https://doi.org/10.1021/acs.analchem.7b03305.

(33) Jackman, J. A.; Saravanan, R.; Zhang, Y.; Tabaei, S. R.; Cho, N.-J. Correlation between Membrane Partitioning and Functional Activity in a Single Lipid Vesicle Assay Establishes Design Guidelines for Antiviral Peptides. Small 2015, 11 (20), 2372-2379. https://doi.org/10.1002/smll.201403638.

(34) Rose, L.; Jenkins, A. T. A. The Effect of the lonophore Valinomycin on Biomimetic Solid Supported Lipid DPPTE/EPC Membranes. Bioelectrochemistry 2007, 70 (2), 387-393. https://doi.org/10.1016/j.bioelechem.2006.05.009.

(35) Hellberg, D.; Scholz, F.; Schauer, F.; Weitschies, W. Bursting and Spreading of Liposomes on the Surface of a Static Mercury Drop Electrode. Electrochem. Commun. 4, 4 (4), 305-309. https://doi.org/10.1016/S1388-2481(02)00279-5.

(36) Hellberg, D.; Scholz, F.; Schubert, F.; Lovrić, M.; Omanović, D.; Hernández, V. A.; Thede, R. Kinetics of Liposome Adhesion on a Mercury Electrode. J. Phys. Chem. B 2005, 109 (30), 14715-14726. https://doi.org/10.1021/jp050816s.

(37) Agmo Hernández, V.; Scholz, F. Kinetics of the Adhesion of DMPC Liposomes on a Mercury Electrode. Effect of Lamellarity, Phase Composition, Size and Curvature of Liposomes, and Presence of the Pore Forming Peptide Mastoparan X. Langmuir 2006, 22 (25), 10723-10731. https://doi.org/10.1021/la060908o.

(38) Agmo Hernández, V.; Niessen, J.; Harnisch, F.; Block, S.; Greinacher, A.; Kroemer, H. K.; Helm, C. A.; Scholz, F. The Adhesion and Spreading of Thrombocyte Vesicles on Electrode Surfaces. Bioelectrochemistry 11, $74 \quad$ (1), 210-216. https://doi.org/10.1016/j.bioelechem.2008.08.003.

(39) Liu, X.; Tong, Y.; Fang, P.-P. Recent Development in Amperometric Measurements of Vesicular Exocytosis. TrAC Trends Anal. Chem. 2019, 113, 13-24. https://doi.org/10.1016/j.trac.2019.01.013.

(40) Fathali, H.; Cans, A.-S. Amperometry Methods for Monitoring Vesicular Quantal Size and Regulation of Exocytosis Release. Pflugers Arch. 2018, 470 (1), 125-134. https://doi.org/10.1007/s00424-017-2069-9.

(41) Hernández, V. A.; Scholz, F. The Electrochemistry of Liposomes. Isr. J. Chem. 2008, 48 (3-4), 169-184. https://doi.org/10.1560/IJC.48.3-4.169.

(42) Laborda, E.; Molina, A.; Batchelor-McAuley, C.; Compton, R. G. Individual Detection and Characterization of Non-Electrocatalytic, Redox-Inactive Particles in Solution by 
Using Electrochemistry. ChemElectroChem 2018, 5 (3), 410-417. https://doi.org/10.1002/celc.201701000.

(43) Andreescu, D.; Kirk, K. A.; Narouei, F. H.; Andreescu, S. Electroanalytic Aspects of Single-Entity Collision Methods for Bioanalytical and Environmental Applications. ChemElectroChem 2018, 5 (20), 2920-2936. https://doi.org/10.1002/celc.201800722.

(44) Liu, Y.; Xu, C.; Yu, P.; Chen, X.; Wang, J.; Mao, L. Counting and Sizing of Single Vesicles/Liposomes by Electrochemical Events. ChemElectroChem 2018, 5 (20), 29542962. https://doi.org/10.1002/celc.201800616.

(45) Fan, F. R. F.; Demaille, C. The Preparation of Tips for Scanning Electrochemical Microscopy; In Scanning Electrochemical Microscopy, 2nd ed.; Mirkin, M. V.; Bard, A. J., Eds.; Marcel Dekker: New York, 2001. https://doi.org/10.1201/b11850-4.

(46) Dick, J. E.; Bard, A. J. Toward the Digital Electrochemical Recognition of Cobalt, Iridium, Nickel, and Iron Ion Collisions by Catalytic Amplification. J. Am. Chem. Soc. 2016, 138 (27), 8446-8452. https://doi.org/10.1021/jacs.6b03202.

(47) Koley, D.; Bard, A. J. Triton X-100 Concentration Effects on Membrane Permeability of a Single HeLa Cell by Scanning Electrochemical Microscopy (SECM). Proc. Natl. Acad. Sci. 2010, 107 (39), 16783-16787. https://doi.org/10.1073/pnas.1011614107.

(48) Keller, C. A.; Kasemo, B. Surface Specific Kinetics of Lipid Vesicle Adsorption Measured with a Quartz Crystal Microbalance. Biophys. J. 1998, 75 (3), 1397-1402. https://doi.org/10.1016/S0006-3495(98)74057-3.

(49) Dimitrievski, K.; Kasemo, B. Simulations of Lipid Vesicle Adsorption for Different Lipid Mixtures. Langmuir 2008, 24 (8), 4077-4091. https://doi.org/10.1021/la703021u.

(50) Dey, S.; Mondal, B.; Dey, A. An Acetate Bound Cobalt Oxide Catalyst for Water Oxidation: Role of Monovalent Anions and Cations in Lowering Overpotential. Phys. Chem. Chem. Phys. 2014, 16 (24), 12221-12227. https://doi.org/10.1039/C4CP01205D.

(51) Ariga, T.; Macala, L. J.; Saito, M.; Margolis, R. K.; Greene, L. A.; Margolis, R. U.; Yu, R. K. Lipid Composition of PC12 Pheochromocytoma Cells: Characterization of Globoside as a Major Neutral Glycolipid. Biochemistry 1988, 27 (1), 52-58. https://doi.org/10.1021/bi00401a010.

(52) Blicher, A.; Wodzinska, K.; Fidorra, M.; Winterhalter, M.; Heimburg, T. The Temperature Dependence of Lipid Membrane Permeability, Its Quantized Nature, and the Influence of Anesthetics. Biophys. J. 2009, $96 \quad$ (11), 4581-4591. https://doi.org/10.1016/j.bpj.2009.01.062.

(53) Wu, H.-L.; Sheng, Y.-J.; Tsao, H.-K. Phase Behaviors and Membrane Properties of Model Liposomes: Temperature Effect. J. Chem. Phys. 2014, 141 (12), 124906. https://doi.org/10.1063/1.4896382.

(54) Masaharu, U.; Shoshin, Y.; Isamu, H. Characteristics of the Membrane Permeability of Temperature-Sensitive Liposome. Bull. Chem. Soc. Jpn. 1991, 64 (5), 1588-1593. https://doi.org/10.1246/bcsj.64.1588.

(55) Cannes, C.; Kanoufi, F.; Bard, A. J. Cyclic Voltammetry and Scanning Electrochemical Microscopy of Ferrocenemethanol at Monolayer and Bilayer-Modified Gold Electrodes. J. Electroanal. Chem. 2003, 547 (1), 83-91. https://doi.org/10.1016/S00220728(03)00192-X.

(56) Cannes, C.; Kanoufi, F.; Bard, A. J. Cyclic Voltammetric and Scanning Electrochemical Microscopic Study of Menadione Permeability through a Self-Assembled Monolayer on a Gold Electrode. Langmuir 2002, 18 (21), 8134-8141. https://doi.org/10.1021/la0258906.

(57) Gennuso, F.; Fernetti, C.; Tirolo, C.; Testa, N.; L'Episcopo, F.; Caniglia, S.; Morale, M. C.; Ostrow, J. D.; Pascolo, L.; Tiribelli, C.; et al. Bilirubin Protects Astrocytes from Its Own Toxicity by Inducing Up-Regulation and Translocation of Multidrug ResistanceAssociated Protein 1 (Mrp1). Proc. Natl. Acad. Sci. U. S. A. 2004, 101 (8), 2470-2475. https://doi.org/10.1073/pnas.0308452100. 


\section{TABLE OF CONTENT GRAPHICAL ABSTRACT}
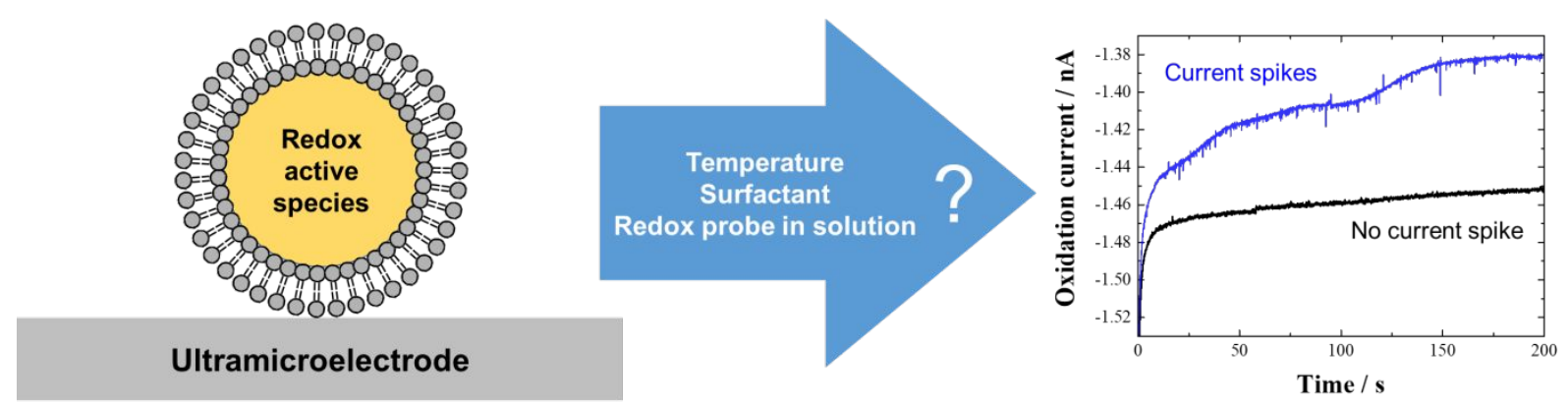